\section{A CLEAR CALL FOR CONSISTENCY: CREATING A WORKING DOCUMENT FOR MANAGEMENT OF DIABETES IN PALLIATIVE CARE PATIENTS}

Madeleine S Turley. St Michael's Hospice, Hereford

10.1136/spcare-2020-PCC.177

Background Diabetes affects a significant proportion of our patients, with a higher prevalence than the UK population $(6 \%)$ due to those with steroid induced and disease related diabetes. This can present both symptomatic challenges and regimen burden, which can require the patient to accept changes that may be at odds with their established management. In the hospice in patient setting a lack of consistency of clinical approach to diabetic patients was observed, due to part time working, a varied medical team and the perceived complexities of diabetic management in this patient group. Whilst a guideline existed, it was held away from the patients" bedside and not consistently reviewed. A new set of 'working documents' was created, based on the existing evidence, held at the patient's bedside. This 2 sided document contains detailed guidance on medical management dependent on prognosis, and incorporates monitoring charts for nursing staff; aiding consistency of approach and clarity of decision making. We audited the use of the form, both before and after education sessions to support its use, with an aim to make any necessary improvements.

Methods We audited 20 diabetes forms both pre and post educations sessions. These involved staff confidence scores pre and post education, and then discussion around the use of the forms.

Results Following the education sessions, the number for forms correctly filled in increased from $\% 5$ to $30 \%$. Confidence scores of staff users also increased significantly from $6.8 / 10$ pre education, to $8.7 / 10$ post education. General feedback was sought and incorporated where appropriate.

Conclusion A clear improvement was noted in the use of the forms following staff educations sessions, which has positively impacted a new, cohesive MDT approach to improving diabetes management.

\section{HAS EMBEDDING SPECIALIST PALLIATIVE CARE IN AN INTERSTITIAL LUNG DISEASE CLINIC IMPACTED ON REFERRALS TO A LOCAL HOSPICE?}

Kym Wakefield, Craig Gouldthorpe, Anne-Marie Bourke, Julie Harper. Marie Curie

\subsection{6/spcare-2020-PCC.178}

Introduction The British Lung Foundation states there should be a clear, standardised pathway to palliative care for patients with Interstitial Lung Disease (ILD). Our regional ILD clinic is based at the Royal Victoria Infirmary in Newcastle upon Tyne. In January 2016, specialist palliative care from the Marie Curie hospice Newcastle was embedded in this clinic. Patients can access same-day palliative care and respiratory reviews. The hospice has a 20-bedded inpatient unit (IPU) and a day therapy unit (DTU).

Aims To evaluate the effect of a collaborative ILD palliative care service on the number of referrals to the Marie Curie hospice, Newcastle.

Methods All patients referred to the hospice between November 2017 and May 2019 with a primary coded diagnosis of a 'non-malignant respiratory condition' were identified using SystmOne. Specific diagnosis, referrer and reason(s) for referral were also collected.

Results A total of 38 patients were identified. The majority of these had a diagnosis of ILD $(n=26)$; the remainder had chronic obstructive pulmonary disease $(n=11)$ or bronchiectasis $(n=1)$. The 26 individuals with ILD accounted for 37 accepted referrals to the hospice (DTU $=21$, IPU=16); some patients were referred more than once. 90\% (19/21) of these DTU referrals originated from the ILD clinic. A wider source of referrals was seen for IPU. The most frequently occurring reasons for referral were breathlessness and cough.

Discussion We are unable to compare current referrals with those prior to 2016 because a different record system was used. However, a search found only one patient with ILD admitted to IPU in 2015. We conclude that this increase is an outcome of our collaborative service. Anecdotal feedback suggests that patients who have met a member of the hospice team in clinic find it easier to accept hospice referral - further research is needed to explore this.

\section{WHAT ARE THE CURRENT TRENDS FOR PATIENTS WITH MOTOR NEURONE DISEASE (MND) ACCESSING HOSPICE INPATIENT UNIT (IPU) SERVICES? A RETROSPECTIVE REVIEW OF ADMISSIONS \& CONSIDERATIONS FOR ONGOING DEVELOPMENT}

Donna Wakefield, Sarah R Hanson, Anna Grundy. Marie Curie Hospice Newcastle

\subsection{6/spcare-2020-PCC.179}

Introduction MND is a progressive neurological disorder with an average prognosis of 2-3 years. NICE guidance recommends that Palliative Care specialists play an integral role in the MDT caring for patients with MND. We aim to examine how patients with MND are accessing the hospice IPU and to consider how we could support them further.

Method Retrospective review of the notes of all patients with MND admitted to hospice IPU over the past 2 years.

Results In the past 24 months, 16/861 (2\%) of admissions to IPU had MND. However, 9/16 of these admissions were for planned carer respite (service no longer available). Excluding planned respite, all admissions (7 admissions, 5 different patients) were through urgent referrals. Three admissions were for social crisis, where one patient experienced problems with their care provider.

For the remaining admissions $(n=4)$, the average age was 71 years, all admitted urgently from home with advanced disease (average 22 months from diagnosis) with rapidly progressive symptoms. Referrals were either from the MND team $(50 \%)$ or the hospice Day Therapy Unit (50\%). There were no referrals from other services such as hospital or GP. Half had some evidence of Advance Care Planning (ACP) documented prior to admission and all had ACP during admission. All patients died, 3 as inpatients and 1 discharged home for end of life care (EOLC). The time between admission to death ranged from 2-40 days. Two patients required withdrawal of life-prolonging treatment; one withdrawal of NIV and one withdrawal of PEG feeding.

Conclusion All patients admitted (excluding planned respite) were referred to hospice IPU urgently. For $80 \%$ this was late in disease and involved EOLC. A new outpatient MND group 